\title{
Ultrasonic assisted direct contact membrane distillation hybrid process for membrane scaling mitigation
}

\author{
Deyin Hou ${ }^{\mathrm{a}, \mathrm{b}, *}$, Zhangxin Wang ${ }^{\mathrm{c}}$, Guoliang Li ${ }^{\mathrm{a}, \mathrm{b}}$, Hua Fan ${ }^{\mathrm{d}}$, Jun Wang ${ }^{\mathrm{a}, \mathrm{b}}$, Hongjing Huang ${ }^{\mathrm{d}}$ \\ a State Key Laboratory of Environmental Aquatic Chemistry, Research Center for Eco-Environmental Sciences, Chinese Academy of Sciences, Beijing 100085, PR China \\ ${ }^{\mathrm{b}}$ Key Laboratory of Drinking Water Science and Technology, Research Center for Eco-Environmental Sciences, Chinese Academy of Sciences, Beijing 100085, PR China \\ c Department of Civil and Environmental Engineering, Vanderbilt University, PMB 51831, 2301 Vanderbilt Place, Nashville, TN 37235-1831, United States \\ d School of Environmental and Chemical Engineering, Nanchang University, Jiangxi 330031, PR China
}

\section{H I G H L I G H T S}

- Ultrasonic assisted membrane distillation hybrid process was designed.

- The effect of ultrasonic irradiation on membrane scaling was investigated.

- Ultrasonic irradiation can effectively mitigate $\mathrm{CaSO}_{4}$ membrane scaling.

- $\mathrm{CaCO}_{3}$ had little effect on permeability with or without ultrasonic irradiation.

- Ultrasonic irradiation can restrain silica colloid deposit and maintain flux

\section{GRA P H I C A L A B S T R A C T}

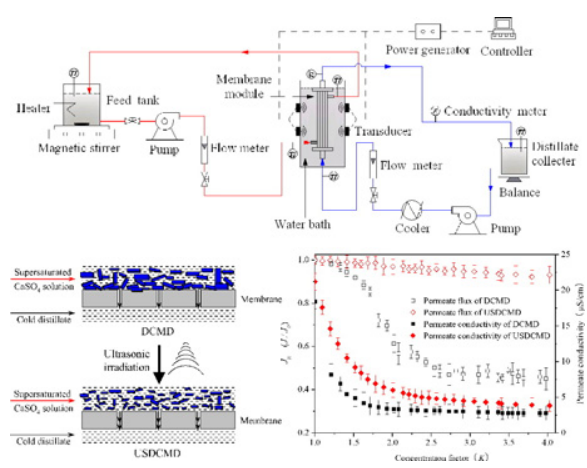

\section{A B S T R A C T}

A novel ultrasonic assisted direct contact membrane distillation hybrid process was designed and the effect of ultrasonic irradiation on membrane scaling mitigation during membrane distillation process was investigated. Under ultrasonic irradiation, ultrasonic wave refreshed the liquid-membrane interface continuously and reduced concentration polarization in the boundary layer adjacent to membrane surface simultaneously. Therefore, ultrasonic irradiation could mitigate membrane scaling caused by $\mathrm{CaSO}_{4}$ and prevented the permeate flux decline. Although the ultrasonic irradiation could restrain the crystal size of $\mathrm{CaCO}_{3}$ deposits and clean membrane surface, the experimental results demonstrated that it was not necessary to introduce ultrasonic irradiation into membrane distillation process for $\mathrm{CaCO}_{3}$ membrane scaling mitigation. Due to fast precipitation rate, the concentration of $\mathrm{CaCO}_{3}$ solution would not cause notable permeate flux decline in the absence of ultrasonic irradiation. There would be a gradual permeate flux decline during the concentration process of silica solution because of the formation and deposition of colloidal polysilicic acid on membrane surface. The ultrasonic irradiation caused the zeta potential of silica colloids to approach neutral and enhanced the tendency of colloid deposit, but the membrane surface could be effectively kept clean and the permeate flux was hardly affected by concentration factor increasing.

(c) 2015 Elsevier B.V. All rights reserved.

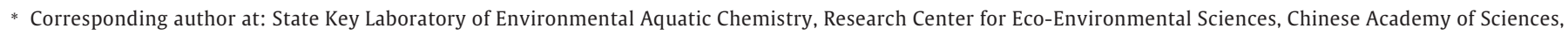
Beijing 100085, PR China.

E-mail address: dyhou@rcees.ac.cn (D. Hou). 


\section{Introduction}

Membrane distillation (MD) is a thermally driven membrane separation technology and usually applied in which water is the major component present in the feed solution to be treated [1]. The MD process may be used as a substitute for conventional separation processes such as multistage vacuum evaporation, reverse osmosis, and distillation [2]. Compared to those processes, the advantages of MD include: (1) lower operating temperature and vapor space required than conventional distillation, (2) lower operating pressure than RO, (3) 100\% (theoretical) rejection of non-volatile solute, (4) unlimited by high osmotic pressure, and (5) lower energy consumption than multistage vacuum evaporation [3-5].

Although there have been extensive studies on the application of MD for water purification, significant advancements are still needed for MD to reach the theoretical cost competitiveness and develop market share growth [6]. Membrane scaling in MD process is of particular importance, as scaling can alter membrane surface properties, change membrane pore structure, potentially lead to wetting of membrane pores and ultimately decrease membrane process efficiency [7-9]. Membrane scaling occurs when inorganic salts precipitate and accumulate on membrane surface, thus blocking the pores for vapor to diffuse across the membrane and subsequently lowering water flux. Scaling of sparingly soluble salts such as $\mathrm{CaCO}_{3}, \mathrm{CaSO}_{4}$, and silicates has been identified as a cause of flux decline when recovering water from natural streams, including brines from desalination processes [10-14].

Recently, many efforts have been made on membrane scaling mitigation during MD process by physical or chemical methods. The $\mathrm{pH}$ control of feed is a common method to reduce or eliminate MD scaling. In almost all cases, acidification of the feed can cause alkaline salts, the main component of scale, to become drastically more soluble [15]. Antiscalants can be used to prevent inorganic scaling, and are potent for carbonate scales, as well as phosphate, sulfate, disperse colloids and metal oxides. He et al. [16] reported that polyacrylic acid antiscalant was particularly effective in reducing calcium sulfate scale during MD process. However, it should be noted that some organic antiscalants may reduce the surface tension of the feed, which can promote membrane wetting. Chen et al. [17] incorporated gas bubbling into direct contact membrane distillation (DCMD), and found that gas bubbling delayed the occurrence of permeate flux decline due to membrane scaling deposition. Gryta [18] performed a study with a magnetic water treatment device on hollow fiber MD, the magnetic field caused significant changes in the morphology of crystal deposits on membrane surface and the $\mathrm{CaCO}_{3}$ deposit layer thickness became smaller, the flux decline was mitigated. The method heating or boiling feed water is called thermal softening, and can also help reduce scale by causing $\mathrm{CaCO}_{3}$ and other salts to precipitate out in heating step [19]. Microfiltration (MF) and nanofiltration (NF) are sometimes used before MD process to remove scaling and large molecules in the feed, Kesieme et al. [20] used a cartridge filter to capture calcium scale and obtained high recovery of groundwater RO concentrate with MD, Karakulski and Gryta [21] introduced NF process to soften the tap water preliminary and improved MD module efficiency.

Ultrasonic wave is referred to the acoustic wave with the frequency between $20 \mathrm{kHz}$ and $10 \mathrm{MHz}$. Several concomitant effects, such as heat generation, mechanics and cavitation effect, present themselves during the propagation of ultrasonic wave in various media, and these effects have been recognized to be beneficial to many physical and chemical processes [22]. For membrane separation processes, the ultrasonic technique is used mainly in membrane fouling monitoring, membrane cleaning, and membrane flux enhancement [23-29]. Li and Mairal et al. [30-32] applied ultrasonic technique as a non-destructive, realtime, in situ measuring technique for direct monitoring of membrane fouling and cleaning during ultrafiltration (UF) and RO, and found that the ultrasonic technique is a useful technique for the non-destructive investigation of fouling and cleaning in membrane applications. Kobayashi et al. [33-37] introduced ultrasonic technique to create novel anti-fouling membrane processes for membrane water treatments, it was reported that ultrasonic irradiation during membrane filtration was very effective in removing foulants from membranes. Massive evidences exist that the ultrasonic effect is useful for water cleaning of fouled membrane, the ultrasonic cleaning presents advantages and is an effective method compared with other typical cleaning methods using physical and chemical methods [38-40].

Although the ultrasonic irradiation has been successfully applied to enhance the performance of membrane separation process such as microfiltration (MF), UF, and RO, relatively few studies have been carried out with the use of ultrasonic to eliminate or prevent the membrane fouling in MD process. Consequently, in the present study, an ultrasonic assisted direct contact membrane distillation (USDCMD) hybrid process was developed to mitigate membrane scaling and enhance the performance of MD process.

\section{Experimental}

\subsection{Materials and membrane module}

The polytetrafluoroethylene (PTFE) hydrophobic hollow fiber membrane with a mean pore diameter of $0.26 \mu \mathrm{m}$, supplied by DD Water Group Co., Ltd. (China), was chosen to fabricate membrane modules. Forty pieces of hollow fibers were assembled into a polyester tube (diameter $d_{\text {in }} / d_{\text {out }}=15 / 20 \mathrm{~mm} / \mathrm{mm}$ ) with two UPVC T-tubes and two ends of the bundle of fibers were sealed with solidified epoxy resin to compose a membrane module. The effective membrane length was $100 \mathrm{~mm}$ for each membrane module. The characteristics of the membrane and membrane modules are presented in Table 1.

\subsection{USDCMD setup}

The USDCMD experimental setup is schematically shown in Fig. 1. The hot feed, which was stirred continually by a magnetic stirrer, flowed through the shell side of the fibers, and the cold distillate flowed through the lumen side. The initial volumes of the feed and the distillate were $4.0 \mathrm{~L}$ and $0.25 \mathrm{~L}$, respectively. Both solutions were circulated in the membrane module with the help of two magnetic pumps (MP-15RN, Shanghai Seisun Pumps, China). The feed and the distillate flowed cocurrently through the module, and the circulation feed rate $\left(V_{f}\right)$ was $0.25 \mathrm{~m} / \mathrm{s}$, while the cold side $\left(V_{p}\right)$ being $1.0 \mathrm{~m} / \mathrm{s}$. The feed temperature $\left(T_{\text {finlet }}\right)$ was fixed at $53^{\circ} \mathrm{C}$ by a Pt- 100 sensor and a heater connected to an external thermostat (XMTD-2202, Yongshang Instruments, China). The distillate temperature $\left(T_{p \text {-inlet }}\right)$ kept at $20{ }^{\circ} \mathrm{C}$ by a spiral glass heat exchanger immersed in the constant temperature trough of the cooler (SDC-6, Nanjing Xinchen Biotechnology, China). The temperature of both fluids was monitored at the inlet and outlet of the membrane module using four Pt-100 thermoresistances connected to a digital meter (Digit RTD, model XMT-808, Yuyao Changjiang Temperature Meter Instruments, China) with an accuracy of $\pm 0.1^{\circ} \mathrm{C}$. An electric conductivity monitor (CM-230A, Shijiazhuang Create Instrumentation Technologies, China) was used to monitor the distillate water quality.

Table 1

The characteristics of the membrane material and the membrane module.

\begin{tabular}{ll}
\hline Membrane and module & Properties \\
\hline Membrane material & PTFE \\
Mean pore diameter $(\mu \mathrm{m})$ & 0.26 \\
Porosity $(\%)$ & 45.07 \\
Inner diameter of hollow fiber $(\mathrm{mm})$ & 0.80 \\
Membrane thickness $(\mathrm{mm})$ & 0.39 \\
Number of hollow fibers & 40 \\
Effective membrane length $(\mathrm{mm})$ & 100 \\
Effective membrane area $\left(\mathrm{cm}^{2}\right)$ & 198.4 \\
\hline
\end{tabular}




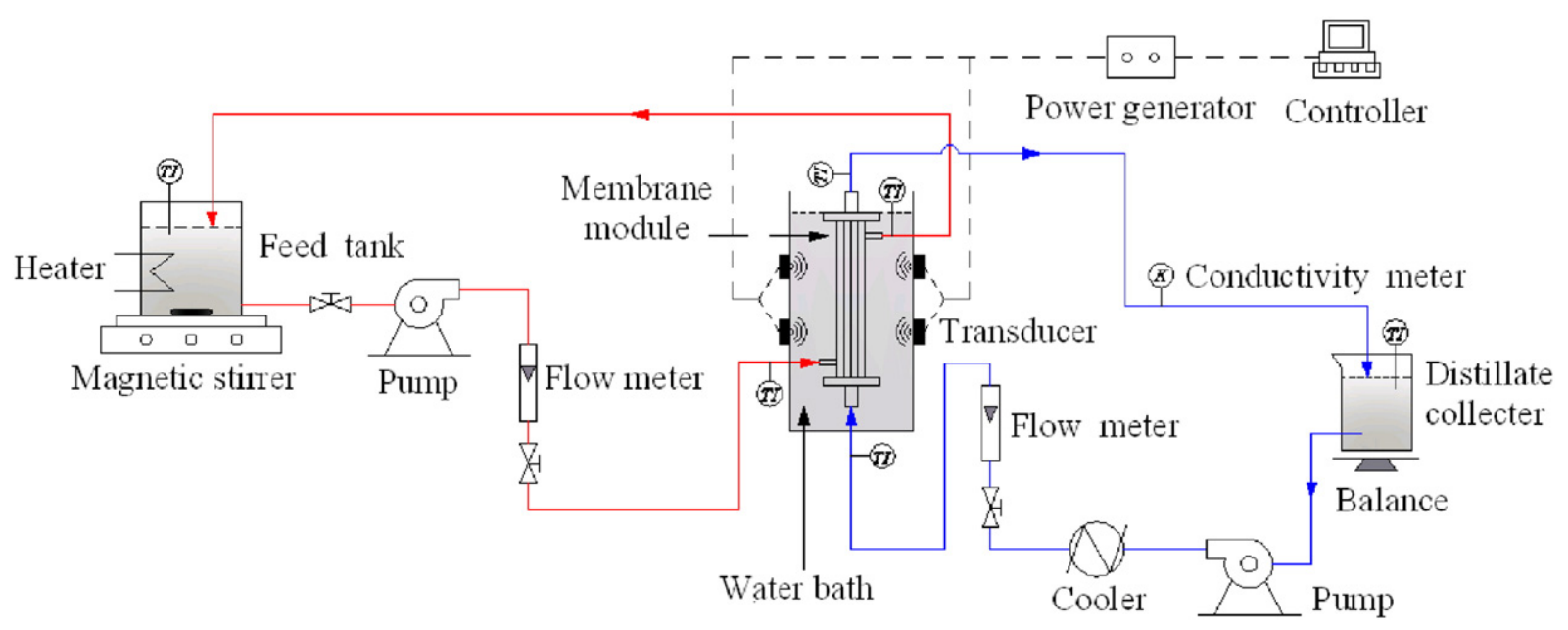

Fig. 1. Schematic diagram of USDCMD system.

In order to investigate the mitigation effect of ultrasonic irradiation on membrane scaling, the membrane module was immersed vertically in a water bath $\left(15 \times 15 \times 42 \mathrm{~cm}^{3}\right)$, transducers were adhered to the four outside surfaces of the water bath stainless steel shell. The ultrasonic bath was capable of generating ultrasonic with a frequency of $20 \mathrm{kHz}$ and an acoustic power of $260 \mathrm{~W}$. The ultrasonic irradiation device was supplied by Quanyi Electronic Equipment Co., Ltd. (Baoding, China).

\subsection{Experimental protocol}

Certified analytical reagent grade $\mathrm{CaSO}_{4}, \mathrm{CaCO}_{3}$, and $\mathrm{Na}_{2} \mathrm{SiO}_{3}$ (Sinopharm Chemical Reagent Co., Ltd. China) were used in this study. The feed solution was prepared by dissolving an appropriate amount of chemical into Milli- $Q$ water. The concentration factor $K$ can be calculated by the following equation:

$K=\frac{Q_{0}}{Q_{0}-Q_{p}}$

where $Q_{0}$ is the initial quantity of feed $(\mathrm{kg}), Q_{p}$ is the cumulative permeate production $(\mathrm{kg})$. The permeate flux $J$ was calculated by the following equation:

$J=\frac{\Delta W}{A \Delta t}$

where $J$ is the permeate flux $\left(\mathrm{kg} / \mathrm{m}^{2} \mathrm{~h}\right), \Delta W$ is the quantity of distillate $(\mathrm{kg}), A$ is the effective area of flat-sheet membrane $\left(\mathrm{m}^{2}\right)$ and $\Delta t$ is the sampling time (h). The relative permeate flux $J_{R}$ was used to describe the change of membrane permeability with the concentration factor increase:

$J_{R}=\frac{J}{J_{0}}$

where $J_{o}$ is the initial permeate flux $\left(\mathrm{kg} / \mathrm{m}^{2} \mathrm{~h}\right)$. When the concentration factor reached 4.0, the MD process was stopped. A new membrane module was used for each experiment in this study. At the completion of each experiment, the membrane was removed from the membrane module for further analysis and excess liquid on the membrane surface was allowed to drain off by gently tilting the hollow fiber.

\subsection{Membrane surface analysis}

The morphology of the scaling layer deposited onto the membrane surface was investigated with a HITACHI S-3000 N scanning electron microscope (SEM) (Hitachi Ltd., Japan). Membrane samples were frozen in liquid nitrogen, fractured to obtain fragments, and sputtered with platinum using a HITACHI E-1010 Ion Sputtering device for SEM observation. The scaled membrane samples were handled gently and without any excessive forces to ensure that the scaling layer remained intact.

\subsection{X-ray diffraction analysis}

Crystallinity properties of the $\mathrm{CaSO}_{4}$ and $\mathrm{CaCO}_{3}$ scales were investigated by an X-ray diffractometer (X'Pert PRO, Panalytical, Holland) at a scanning rate of $4^{\circ} / \mathrm{min}$. The angle of diffraction $(2 \theta)$ was varied from $10^{\circ}$ to $80^{\circ}$ using a step size of $0.02^{\circ}$. X-ray tube voltage and current were set at $40 \mathrm{kV}$ and $40 \mathrm{~mA}$, respectively.

\subsection{Zeta potential measurement}

The measurements of zeta potential of silica colloid were conducted at a fixed temperature of $53{ }^{\circ} \mathrm{C}$ to operate within controllable laboratory conditions. The zeta potential was determined with a Zetasizer 2000 zeta potential analyzer (Malvern Co., Malvern Town, U.K.). For each zeta potential measurement, about $1.5 \mathrm{~mL}$ of the silica solution with different concentration factor was poured into a $4.0 \mathrm{~mL}$ plastic cuvette, then a palladium electrode pair was immersed in the tested sample and the zeta potential was obtained by measuring at least three single

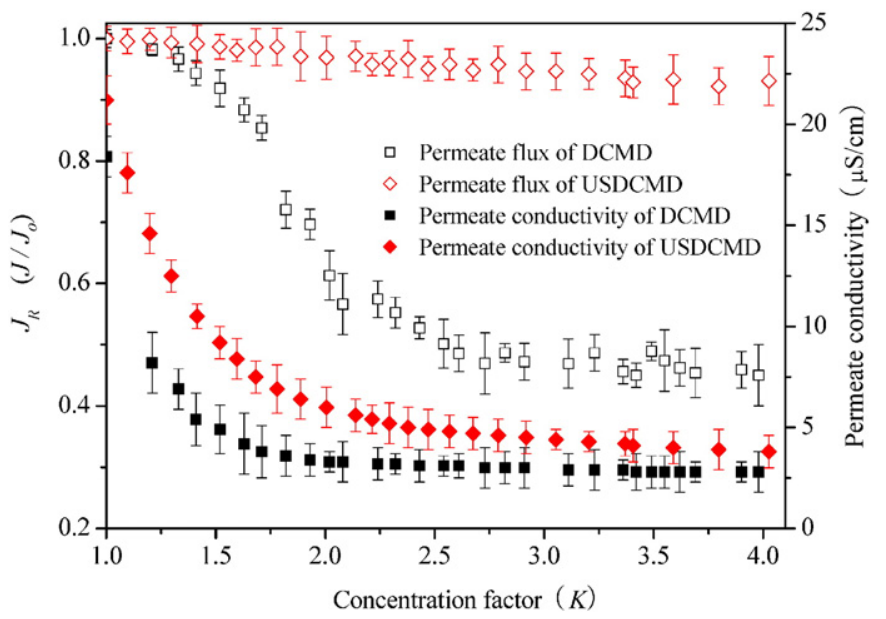

Fig. 2. Effect of ultrasound irradiation on the process of DCMD concentration of $\mathrm{CaSO}_{4}$ solution. 


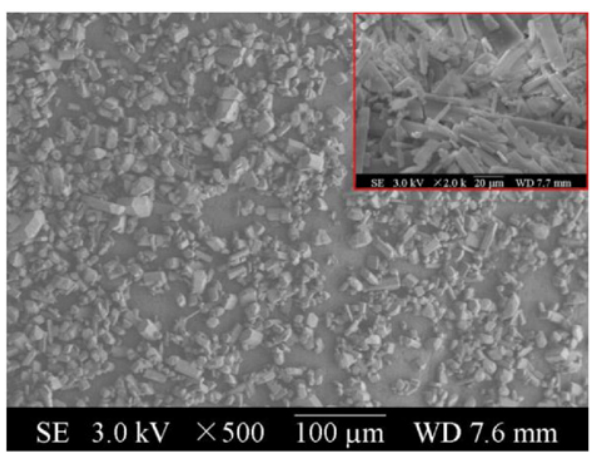

(a)

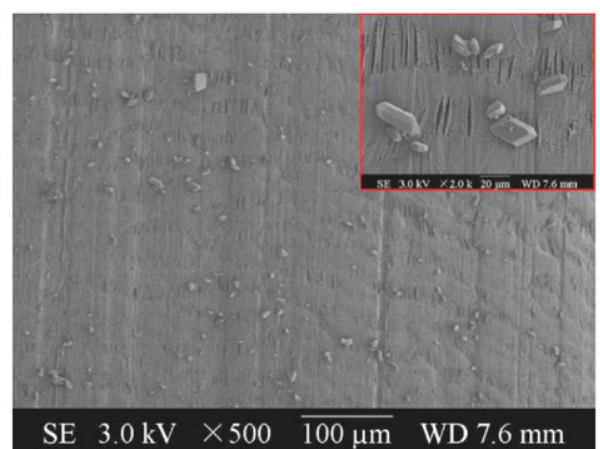

(b)

Fig. 3. Morphological image of the fouled PTFE membrane with $\mathrm{CaSO}_{4}$ : (a) in the absence of ultrasound and (b) in the presence of ultrasound.

samples. After each measurement, the samples were poured back to the feed tank to guarantee the concentration factor accuracy.

\section{Results and discussions}

\subsection{Concentration of $\mathrm{CaSO}_{4}$ solution}

The solution containing $2000 \mathrm{mg} / \mathrm{L} \mathrm{CaSO}_{4}$ was tested for concentration in the presence and absence of ultrasonic irradiation. The permeate flux as a function of concentration factor is illustrated in Fig. 2.

The initial concentration of $\mathrm{CaSO}_{4}$ in the feed solution was near to the saturation limit. Because inorganic salt cannot be transported through the hydrophobic membrane, the solution became supersaturated as the experiment progressed, causing the precipitation of $\mathrm{CaSO}_{4}$. As can be seen from Fig. 2, the higher concentration factor resulted in a lower permeate flux for DCMD process. When the concentration factor reached 4.0 , the permeate flux declined about $55 \%$ compared with the initial permeate flux. The microstructure of the PTFE hollow fiber membrane fouled with the $\mathrm{CaSO}_{4}$ scaling is shown in Fig. 3. It can be found that most of the membrane surface was covered with a layer of $\mathrm{CaSO}_{4}$, which was the reason for the sharp decrease in the permeate flux observed during the concentration of the $\mathrm{CaSO}_{4}$ solution in the absence of ultrasonic irradiation.

The permeate flux increased markedly in the presence of ultrasonic irradiation as indicated in Fig. 2, while the rejection values of the hydrophobic membrane kept almost $100 \%$. The higher the concentration

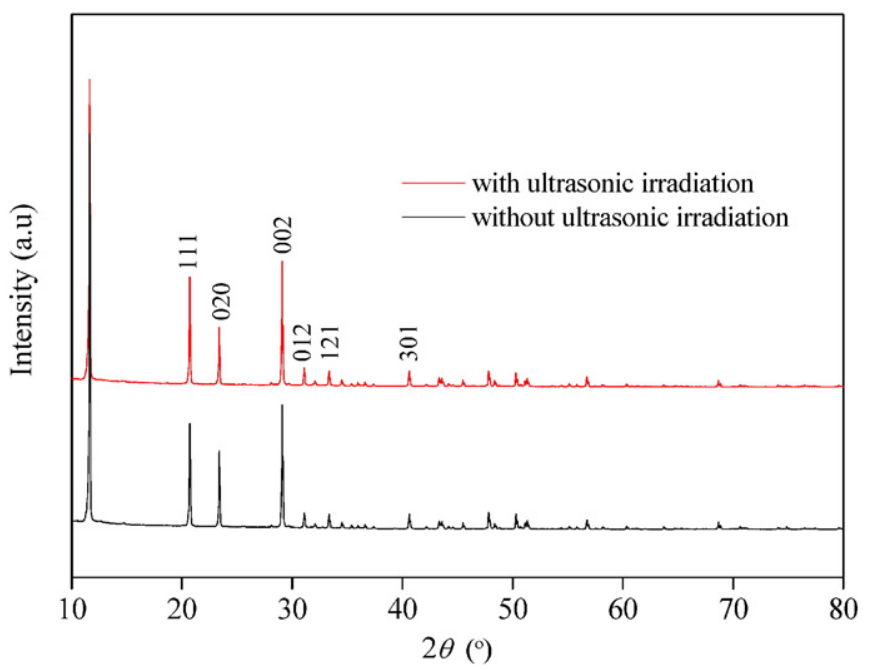

Fig. 4. XRD patterns of $\mathrm{CaSO}_{4}$ scales (a.u., arbitrary unit). factor was, the more obvious the ultrasonic enhancement of permeate flux could be obtained. As can be seen from Fig. 2, the relative permeate flux maintained $93 \%$ when the concentration factor reached 4.0 in the presence of ultrasonic irradiation. The ultrasonic irradiation can effectively mitigate the membrane scaling caused by the sparingly soluble salt $\mathrm{CaSO}_{4}$, which can also be observed from images of the morphology of the membrane surfaces. The difference in the surface morphologies of the membranes fouled with $\mathrm{CaSO}_{4}$ crystals in the absence and presence of ultrasonic irradiation is shown in Fig. 3. For the membrane used in USDCMD process, although there were still some salt crystals deposited on the membrane surface, the quantity of the $\mathrm{CaSO}_{4}$ crystals was less and the crystal size became much smaller compared with those deposited on the surface of the membrane without ultrasonic irradiation. Crystal structures of the $\mathrm{CaSO}_{4}$ scales are shown in Fig. 4, given by the $\mathrm{X}$-ray diffraction (XRD) analysis. It can be found that the ultrasonic irradiation had no perceptible effects on crystallinity properties of the $\mathrm{CaSO}_{4}$ scale. Both of the $\mathrm{CaSO}_{4}$ scales presented monoclinic crystals with or without ultrasonic irradiation. The strongest peak near $2 \theta$ of $11.5^{\circ}$ was observed in the XRD pattern, which implied that the scales were mainly composed of $\mathrm{CaSO}_{4} \cdot 2 \mathrm{H}_{2} \mathrm{O}$.

Ultrasonic irradiation could mitigate membrane scaling caused by $\mathrm{CaSO}_{4}$ and prevent the permeate flux declining without obvious loss in membrane rejection, this could be attributed to ultrasonic cleaning of the membrane surface. The schematic illustration of the ultrasonic irradiation effect on mitigation of $\mathrm{CaSO}_{4}$ scaling deposition on membrane surface was given in Fig. 5. Ultrasonic wave can bring significant mechanical and thermal effects, and generate powerful shock wave

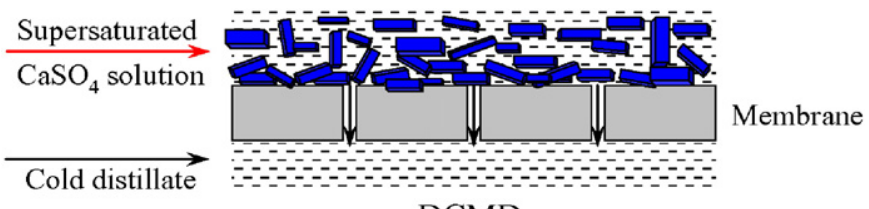

DCMD

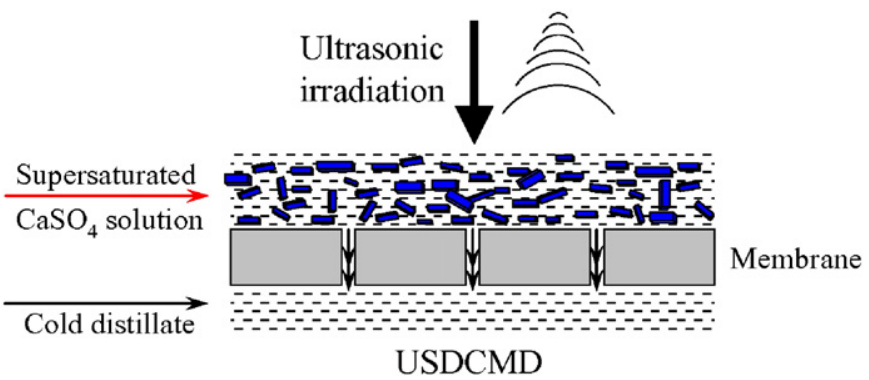

Fig. 5. Schematic illustration of ultrasonic irradiation effect on mitigation of $\mathrm{CaSO}_{4}$ scaling. 


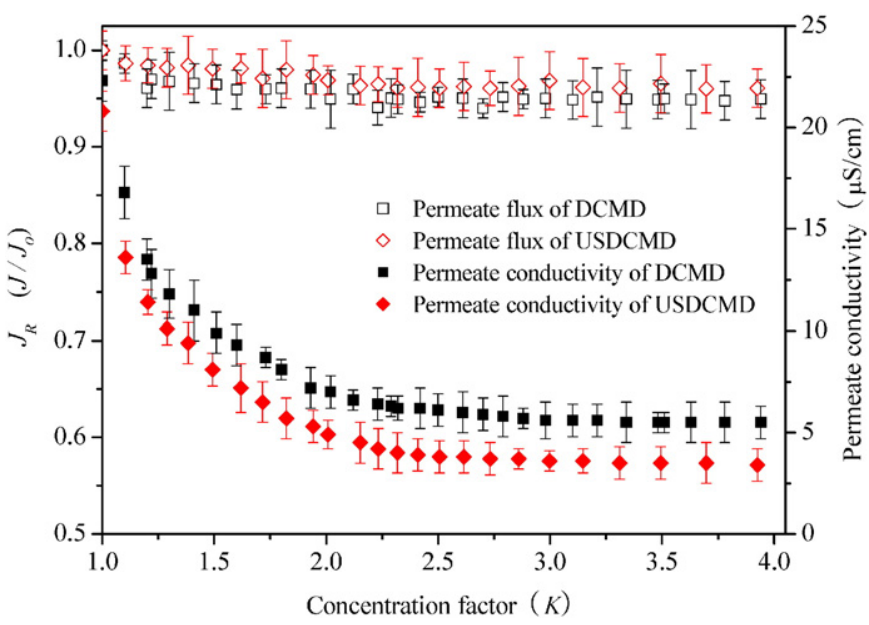

Fig. 6. Effect of ultrasound irradiation on the process of DCMD concentration of $\mathrm{CaCO}_{3}$ solution.

and microstreaming with high speed. The microstreaming, shock wave and acoustic vortex streaming can continuously stimulate the liquidmembrane interface, therefore refreshed the interface and prevented the deposition of $\mathrm{CaSO}_{4}$ crystals. In addition, the mechanical effect promoted turbulence, which reduced the temperature polarization and concentration polarization. As a result, the lower concentration in the boundary layer also reduced the likelihood of crystallization.

\subsection{Concentration of $\mathrm{CaCO}_{3}$ solution}

The concentration processes of calcium carbonate supersaturated solution through MD were investigated with the initial $\mathrm{CaCO}_{3}$ concentration of $100 \mathrm{mg} / \mathrm{L}$. The influence of ultrasonic irradiation on the performance of PTFE hydrophobic membrane is shown in Fig. 6.

The insoluble salt $\mathrm{CaCO}_{3}$ did not cause notable flux decline with the concentration factor increase, which was consistent with the finding of He et al. [12]. It was reported that the negligible negative effect of membrane scaling caused by a pure $\mathrm{CaCO}_{3}$ solution on permeate flux can be attributed to the fast precipitation rate of $\mathrm{CaCO}_{3}$, which caused the formation of fine $\mathrm{CaCO}_{3}$ particles [41]. The fine particles would suspend in the bulk solution rather than depositing on the membrane surface. Although the SEM analysis at the conclusion of the DCMD experiment revealed the presence of some salt crystals scattered on the membrane surface as shown in Fig. 7, these salt crystals did not completely cover the entire membrane surface area. On the other hand, in the Fig. 7, it can be found that the aggregates of the $\mathrm{CaCO}_{3}$ salt crystals were very loose, and this was also beneficial to maintain the permeate flux. When the concentration factor reached 4.0, the relative permeate flux was about 95\%, the permeate flux declined only 5\% compared with the initial permeate flux.

In the presence of ultrasonic irradiation, the feed solution became cloudy almost immediately, which indicated that ultrasonic irradiation accelerated the crystallization rate of calcium carbonate. Owing to ultrasonic irradiation, the nucleation induction period of calcium carbonate was shortened, a large number of crystal nuclei formed rapidly and the crystal size decreased. Moreover, the powerful shock wave and microstreaming with high speed accompanied by ultrasonic irradiation also restrained the formation of large size calcium carbonate crystal aggregates in some extent. In Fig. 7, it can be found that there was a small quantity of fine calcium carbonate crystals scattered on the membrane surface in the presence of ultrasonic irradiation. In addition, it should be noted that ultrasonic irradiation could not clean the entire membrane surface, and some deposition of $\mathrm{CaCO}_{3}$ could have occurred as a result of hot spots created within the liquid during ultrasonic irradiation, as the solubility of $\mathrm{CaCO}_{3}$ was lowered with an increase in temperature. Fig. 8 presents the XRD patterns of the $\mathrm{CaCO}_{3}$ scales with or without ultrasonic irradiation. Being similar to the USDCMD process of calcium sulfate solution, the ultrasonic irradiation hardly affected crystallinity properties of the $\mathrm{CaCO}_{3}$ scale. The main characteristic planes of (012), (104), (110), (113), (202), (018), (116), (1010), (214), and (300), corresponding to $2 \theta$ value of $23.1^{\circ}, 29.3^{\circ}, 35.9^{\circ}, 39.3^{\circ}, 43.1^{\circ}$, $47.4^{\circ}, 48.4^{\circ}, 57.3^{\circ}, 60.6^{\circ}$, and $64.6^{\circ}$, respectively, appeared in all patterns and indicated a calcite structure. The sharp crystalline peaks demonstrated highly crystallized calcite phase with or without ultrasonic irradiation.

\subsection{Concentration of silica solution}

Silicate stock solution was prepared from sodium metasilicate and stored in a polyethylene bottle. The supersaturated silica solution can be obtained with adding known volumes of silicate stock solution and water in the polyethylene container. The silica solution concentration was $150 \mathrm{mg} / \mathrm{L}$ expressed as $\mathrm{SiO}_{2}$, and its $\mathrm{pH}$ was adjusted to $7.0 \mathrm{using} \mathrm{di}-$ lute hydrochloric acid before MD experiment starting. The permeate flux as a function of concentration factor is presented in Fig. 9.

There was a gradual permeate flux decline with concentration factor increasing during DCMD operation. When the concentration factor reached 4.0 , the permeate flux declined about 20\% compared with the initial permeate flux. Silica exists in many crystalline and amorphous forms. Crystalline silica has a very low solubility in water (on the order of $6 \mathrm{mg} / \mathrm{L} \mathrm{SiO}_{2}$ ). By contrast, amorphous silica has a much higher solubility in the range of 100 to $140 \mathrm{mg} / \mathrm{L} \mathrm{SiO}_{2}$. The potential for silica scaling increases when the dissolved silica in an aqueous system exceeds the amorphous silica solubility limit. When dissolved in water, silica forms silicic acid. During the concentration process of silica solution, the silicic acid underwent base-catalyzed polymerization leading to the formation and deposition of colloidal polysilicic acid on membrane

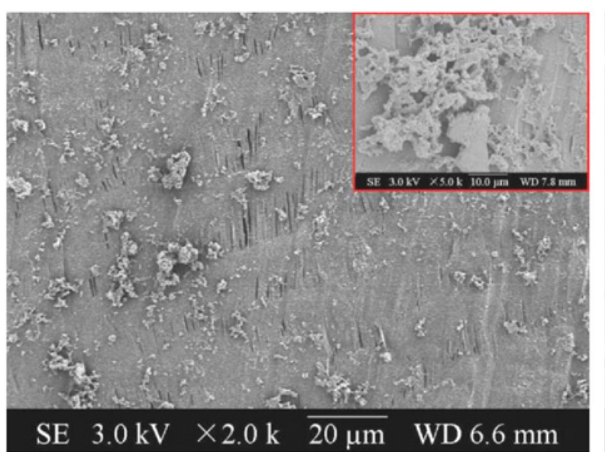

(a)

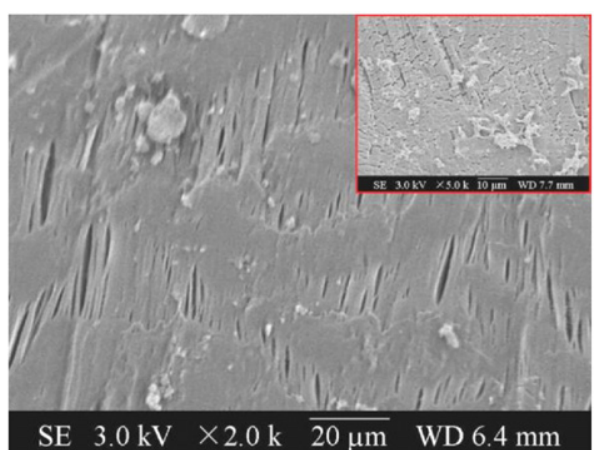

(b) 


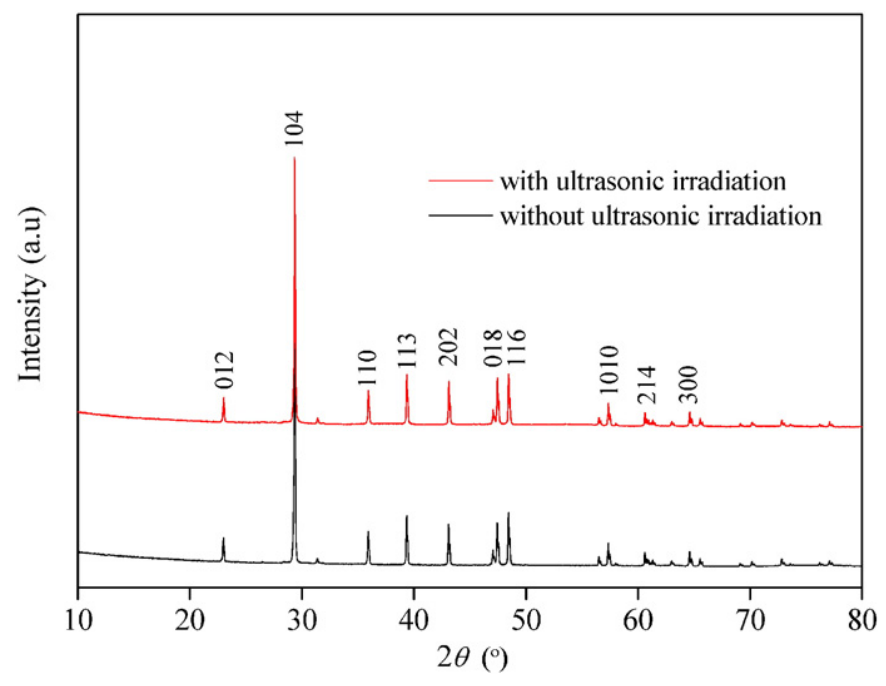

Fig. 8. XRD patterns of $\mathrm{CaCO}_{3}$ scales (a.u., arbitrary unit).

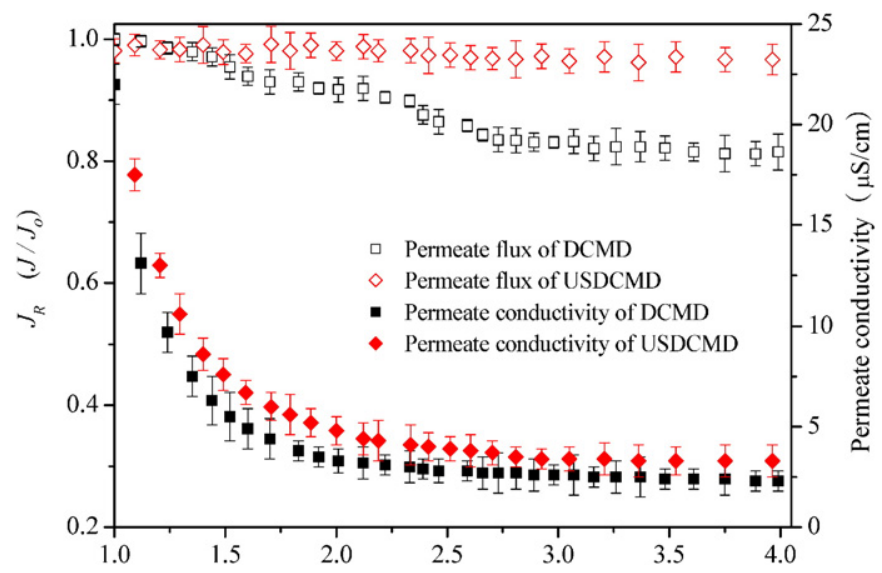

Fig. 9. Effect of ultrasound irradiation on the process of DCMD concentration of $\mathrm{Na}_{2} \mathrm{SiO}_{3}$ solution.

surfaces. The deposited colloids would form a gel layer and cover part of membrane surface, which subsequently led to permeate flux decline. The images of the PTFE membrane fouled with the $\mathrm{SiO}_{2}$ scaling at the end of the experiment are shown in Fig. 10, and the SEM analysis confirmed an amorphous silica scaling layer on the membrane surface.
The permeate flux was hardly affected by concentration factor increasing in the presence of ultrasonic irradiation as illustrated in Fig. 9. When the concentration factor reached 4.0 , the relative permeate flux declined a little and maintained about 97\%. The ultrasonic irradiation can more or less influence the properties of the silica solution during the USDCMD process. As shown in Fig. 11, it can be found that the zeta potential of the silica colloid without ultrasonic irradiation was more negative than that of the silica colloid in the presence of ultrasonic irradiation. In general, the more negative or more positive zeta potentials will cause colloid particles to repel each other due to same charges, and remain colloid system stable and dispersed. On the contrary, as the zeta potential approaches neutral, particles tend to aggregate and deposit on membrane surface. Although the ultrasonic irradiation caused the zeta potential of silica colloids approach neutral and enhanced the tendency of colloid deposit, it should be noted that the surface of the PTFE membrane used in USDCMD process was much cleaner compared with that of the PTFE membrane without ultrasonic irradiation as shown in Fig. 10. The experimental results demonstrated that the ultrasonic irradiation can still effectively mitigate the membrane scaling caused by silica colloids despite the enhancement of the colloid deposit tendency because of the powerful shock wave and microstreaming with high speed accompanied by ultrasonic irradiation.

\section{Conclusions}

In the present work, an ultrasonic assisted direct contact membrane distillation hybrid process was designed and the influence of ultrasonic irradiation on membrane scaling mitigation during DCMD process was investigated.

Under ultrasonic irradiation, the higher the concentration factor of $\mathrm{CaSO}_{4}$ solution was, the more obvious the ultrasonic enhancement of permeate flux could be observed. Ultrasonic wave brought significant mechanical and thermal effects, and generate powerful shock wave and microstreaming with high speed. The mechanical effect promoted turbulence, reduced the concentration polarization in the boundary layer adjacent to membrane surface. The microstreaming, shock wave and acoustic vortex streaming stimulated the liquid-membrane interface continuously, therefore refreshed the interface and prevented the deposition of $\mathrm{CaSO}_{4}$ crystals.

Although the ultrasonic irradiation could restrain the crystals size of $\mathrm{CaCO}_{3}$ deposits and clean membrane surface, it was not necessary to introduce ultrasonic irradiation into DCMD process for the $\mathrm{CaCO}_{3}$ membrane scaling mitigation and permeate flux maintenance. Due to fast precipitation rate, the insoluble salt $\mathrm{CaCO}_{3}$ precipitated in the bulk solution rather than on the membrane surface, and the concentration of the calcium carbonate solution did not cause notable permeate flux decline in the absence of ultrasonic irradiation.

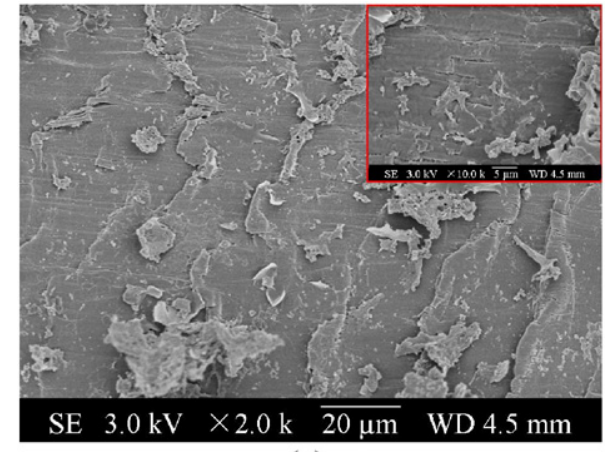

(a)

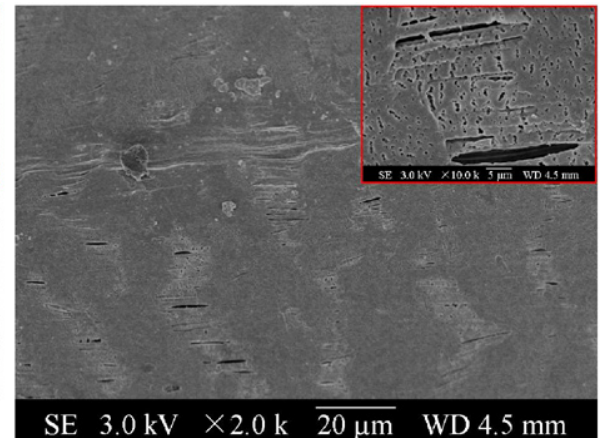

(b)

Fig. 10. Morphological image of the fouled PTFE membrane with silica: (a) in the absence of ultrasound and (b) in the presence of ultrasound. 


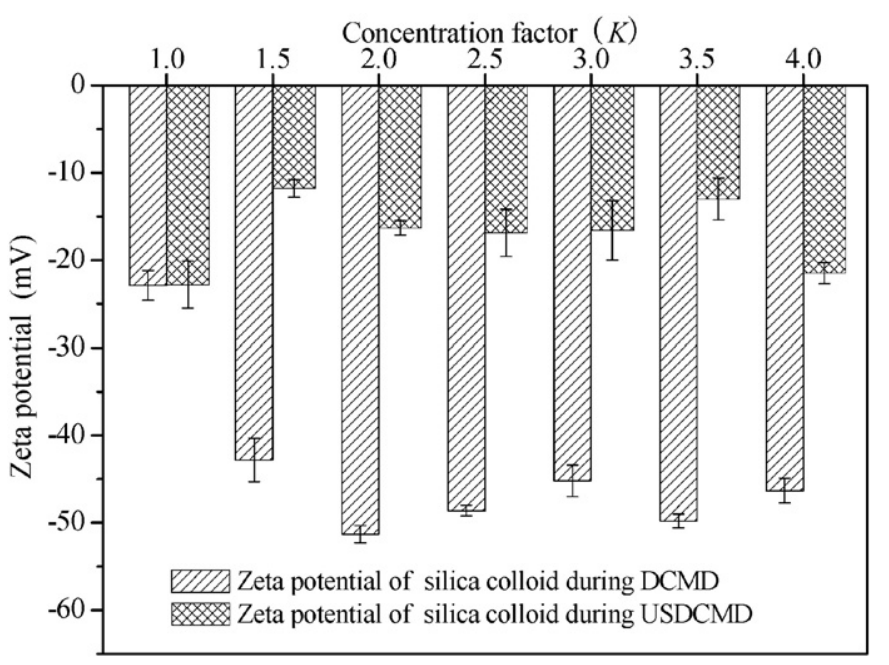

Fig. 11. Effect of ultrasound irradiation on the zeta potential of the silica colloids.

There would be a gradual permeate flux decline during the concentration process of silica solution because of the formation and deposition of colloidal polysilicic acid on membrane surfaces. Although the ultrasonic irradiation caused the zeta potential of silica colloids to approach neutral and enhanced the tendency of colloid deposit, the membrane surface could be effectively kept clean and the permeate flux was hardly affected by concentration factor increasing.

\section{Acknowledgments}

Financial support provided by the National Natural Science Foundation of China (No. 51478454, 51138008) and the special fund from the State Key Laboratory of Environmental Aquatic Chemistry (No. 13Z04ESPCT) are gratefully acknowledged.

\section{References}

[1] K.Y. Wang, T.S. Chung, M. Gryta, Hydrophobic PVDF hollow fiber membranes with narrow pore size distribution and ultra-thin skin for the fresh water production through membrane distillation, Chem. Eng. Sci. 63 (2008) 2587-2589.

[2] K.W. Lawson, D.R. Lloyd, Membrane distillation, J. Membr. Sci. 124 (1997) 1-25.

[3] A. Chafidz, S. Al-Zahrani, M.N. Al-Otaibi, C.F. Hoong, T.F. Lai, M. Prabu, Portable and integrated solar-driven desalination system using membrane distillation for arid remote areas in Saudi Arabia, Desalination 345 (2014) 36-49.

[4] C.R. Martinetti, A.E. Childress, T.Y. Cath, High recovery of concentrated RO brines using forward osmosis and membrane distillation, J. Membr. Sci. 331 (2009) 31-39.

[5] E. El-Zanati, K.M. El-Khatib, Integrated membrane-based desalination system, Desalination 205 (2007) 15-25.

[6] M.M. Teoh, T.S. Chung, Membrane distillation with hydrophobic macrovoid-free PVDF-PTFE hollow fiber membranes, Sep. Purif. Technol. 66 (2009) 229-236.

[7] D.M. Warsinger, J. Swaminathan, E. Guillen-Burrieza, H.A. Arafat, J.H. Lienhard, Scaling and fouling in membrane distillation for desalination applications: a review, Desalination 356 (2015) 294-313.

[8] H. Susanto, Towards practical implementations of membrane distillation, Chem. Eng. Process. 50 (2011) 139-150.

[9] S. Shirazi, C.J. Lin, D. Chen, Inorganic fouling of pressure-driven membrane processes-a critical review, Desalination 250 (2010) 236-248.

[10] K.L. Hickenbottom, T.Y. Cath, Sustainable operation of membrane distillation for enhancement of mineral recovery from hypersaline solutions, J. Membr. Sci. 454 (2014) 426-435

[11] E. Curcio, X. Ji, G. Di Profio, S. Al Obaidani, E. Fontananova, E. Drioli, Membrane distillation operated at high seawater concentration factors: role of the membrane on $\mathrm{CaCO}_{3}$ scaling in presence of humic acid, J. Membr. Sci. 346 (2010) 263-269.

[12] F. He, K.K. Sirkar, J. Gilron, Studies on scaling of membranes in desalination by direct contact membrane distillation: $\mathrm{CaCO}_{3}$ and mixed $\mathrm{CaCO}_{3} / \mathrm{CaSO}_{4}$ systems, Chem. Eng. Sci. 64 (2009) 1844-1859.
[13] M. Gryta, Fouling in direct contact membrane distillation, J. Membr. Sci. 325 (2008) 383-394.

[14] J.P. Mericq, S. Laborie, C. Cabassud, Vacuum membrane distillation of seawater reverse osmosis brines, Water Res. 44 (2010) 5260-5273.

[15] L.D. Tijing, Y.C. Woo, J.S. Choi, S. Lee, S.H. Kim, H.K. Shon, Fouling and its control in membrane distillation-a review, J. Membr. Sci. 475 (2015) 215-244.

[16] F. He, K.K. Sirkar, J. Gilron, Effects of antiscalants to mitigate membrane scaling by direct contact membrane distillation, J. Membr. Sci. 345 (2009) 53-58.

[17] G.Z. Chen, X. Yang, R. Wang, A.G. Fane, Performance enhancement and scaling control with gas bubbling in direct contact membrane distillation, Desalination 308 (2013) 47-55.

[18] M. Gryta, The influence of magnetic water treatment on $\mathrm{CaCO}_{3}$ scale formation in membrane distillation process, Sep. Purif. Technol. 80 (2011) 293-299.

[19] M. Gryta, Desalination of thermally softened water by membrane distillation process, Desalination 257 (2010) 30-35.

[20] U.K. Kesieme, N. Milne, H. Aral, C.Y. Cheng, M. Duke, Economic analysis of desalination technologies in the context of carbon pricing, and opportunities for membrane distillation, Desalination 323 (2013) 66-74.

[21] K. Karakulski, M. Gryta, Water demineralisation by NF/MD integrated processes, Desalination 177 (2005) 109-119.

[22] L.Y. Liu, Z.W. Ding, L.J. Chang, R.Y. Ma, Z.R. Yang, Ultrasonic enhancement of membrane-based deoxygenation and simultaneous influence on polymeric hollow fiber membrane, Sep. Purif. Technol. 56 (2007) 133-142.

[23] C. Zhua, G.L. Liu, C.S. Cheung, C.W. Leung, Z.C. Zhu, Ultrasonic stimulation on enhancement of air gap membrane distillation, J. Membr. Sci. 161 (1999) 85-93.

[24] J.H. Chen, Y.C. Yang, J.Y. Huang, On-line monitoring and diagnosis of membrane fouling using ultrasonic techniques, Chemom. Intell. Lab. Syst. 127 (2013) 147-157.

[25] R. Sanderson, J.X. Li, L.J. Koen, L. Lorenzen, Ultrasonic time-domain reflectometry as a non-destructive instrumental visualization technique to monitor inorganic fouling and cleaning on reverse osmosis membranes, J. Membr. Sci. 207 (2002) 105-117.

[26] J.X. Li, R.D. Sanderson, E.P. Jacobs, Ultrasonic cleaning of nylon microfiltration membranes fouled by Kraft paper mill effluent, J. Membr. Sci. 205 (2002) 247-257.

[27] D. Chen, L.K. Weavers, H.W. Walker, J.J. Lenhart, Ultrasonic control of ceramic membrane fouling caused by natural organic matter and silica particles, J. Membr. Sci. 276 (2006) 135-144.

[28] A. Mirzaie, T. Mohammadi, Effect of ultrasonic waves on flux enhancement in microfiltration of milk, J. Food Eng. 108 (2012) 77-86.

[29] M. Cai, S.N. Zhao, H.H. Liang, Mechanisms for the enhancement of ultrafiltration and membrane cleaning by different ultrasonic frequencies, Desalination 263 (2010) 133-138.

[30] X.H. Li, J.X. Li, J. Wang, H. Wang, B.Q. He, H.W. Zhang, W.S. Guo, H.H. Ngo, Experimental investigation of local flux distribution and fouling behavior in double-end and dead-end submerged hollow fiber membrane modules, J. Membr. Sci. 453 (2014) $18-26$

[31] A.P. Mairal, A.R. Greenberg, W.B. Krantz, Investigation of membrane fouling and cleaning using ultrasonic time-domain reflectometry, Desalination 130 (2000) 45-60.

[32] J.X. Li, R.D. Sanderson, In situ measurement of particle deposition and its removal in microfiltration by ultrasonic time-domain reflectometry, Desalination 146 (2002) $169-175$.

[33] M.L. Xu, X.H. Wen, X. Huang, Z.Y. Yu, M. Zhu, Mechanisms of membrane fouling controlled by online ultrasound in an anaerobic membrane bioreactor for digestion of waste activated sludge, J. Membr. Sci. 445 (2013) 119-126.

[34] S. Muthukumaran, S. Kentish, S. Lalchandani, M. Ashokkumar, R. Mawson, G.W. Stevens, F. Grieser, The optimisation of ultrasonic cleaning procedures for dairy fouled ultrafiltration membranes, Ultrason. Sonochem. 12 (2005) 29-35.

[35] T. Kobayashi, T. Kobayashi, Y. Hosaka, N. Fujii, Ultrasound-enhanced membranecleaning processes applied water treatments: influence of sonic frequency on filtration treatments, Ultrasonics 41 (2003) 185-190.

[36] M.F.N. Secondes, V. Naddeo, V. Belgiorno, F.B. Jr, Removal of emerging contaminants by simultaneous application of membrane ultrafiltration, activated carbon adsorption, and ultrasound irradiation, J. Hazard. Mater. 264 (2014) 342-349.

[37] M.L. Xu, X.H. Wen, Z.Y. Yu, Y.S. Li, X. Huang, A hybrid anaerobic membrane bioreactor coupled with online ultrasonic equipment for digestion of waste activated sludge, Bioresour. Technol. 102 (2011) 5617-5625.

[38] C. Loderer, D. Pawelka, W. Vatier, P. Hasal, W. Fuchs, Dynamic filtration-ultrasonic cleaning in a continuous operated filtration process under submerged conditions, Sep. Purif. Technol. 119 (2013) 72-81.

[39] S.R. Gonzalez-Avila, F. Prabowo, A. Kumar, C.D. Ohl, Improved ultrasonic cleaning of membranes with tandem frequency excitation, J. Membr. Sci. 415-416 (2012) 776-783.

[40] S. Muthukumaran, K. Yang, A. Seuren, S. Kentish, M. Ashokkumar, G.W. Stevens, F. Grieser, The use of ultrasonic cleaning for ultrafiltration membranes in the dairy industry, Sep. Purif. Technol. 39 (2004) 99-107.

[41] L.D. Nghiem, T. Cath, A scaling mitigation approach during direct contact membrane distillation, Sep. Purif. Technol. 80 (2011) 315-322. 